des automotrices actiulles à courant monophasé, comprendra des voitures mixtes de tre ot ze classes à couloir latéral. Les nouvelles voitures seront de 3 e classe, à couloir central et à caisse entièrement métallique.

Les quatre moteurs de chaque automolrice, d'une puissance continue de 125 chevaux, sont suspendus par le nez.

Ils attaquent l'essieu au moyen d'une engrenage fixé non sur l'essieu mais sur le centre de la rone.

Les deux moleurs dun mème bogie sonl couplés en série of alimentés sous la tension totale de 1.500 volts, soit 750 volts par moteur. Ils sont à refroidissement naturel. La pleine puissance est oblemue a la vitesse de 45 kilometres I $^{\prime}$ heure el peut tre maintenue jusqu'a 55 kilometres a Theure, par lo shuntage des inducleurs.

Les automotrices sonl munies du controble à unités multiples.

Le mêcanisme de contròle, du type sêrie parallele, consiste un contacteurs actionnes par un arbre à cames.

Elles sont munies en outre d'un frein rhéostatique leur permeltant dexercer un effort de retenue dans les descentes.

Les locomolives destinées aux trains de marchandises et aux trains de vorageurs ommibus sont également à deux bogies moleurs a adherence totale et armées de 4 moteurs suspendus par le nez. Leur puissance continue est de 1.000 chevaux et la puissance unihoraire de 1.400 chevaux. Les locomotives à vovageurs peuvent atteindre la vitesse de 80 kilomètres à l'heure, celles à marchandises la vilesse de 60 kilomètres. Ces machines ne different d'ailleurs entre elles que par le rapport d'engrenage.

Les deux bogies sont atteles entre eux et portent les appareils de traction el de tamponnement. Les efforls de traction et de choc sont ainsi transmis indépendamment de la caisse. Comme conse̊quence de cette disposition, le pivot d'un des bogies comporte un déplacement longitudinal.

Pour obtenir une longue pẻriode doscillation latêrale de la aisst sur les bogies et diminuer ainsi rinkensite des chocs sur la voie dans les entrées en courbes, la surface d'appui des pivots est concave vers le sol, de telle sorte que la caisse oscille autour d'un axe longitudinal situé à peu près dans le plan des essieux, c'est-à-dire très bas. La stabilité de la caisse est assurée au moyen de 4 équilibreurs latéraux à ressorts réunissant la caisse aux châssis des bogies qui n'ont plus, par conséquent, de patins latéraux. Ces équilibres sont construits de manière à n'offrir aucune résistance à la rotation horizontale des bogies.

Chaque moteur transmet son effort à l'essieu correspondant au moyen de 2 roues dentées fixées sur les centres de roues.

Les moteurs sont alimentés directement à la tension de 1.500 volts. Leur puissance continue est de 250 chevaux et la puissance unihoraire de 350 chevaux. Ils sont ventilés artificiellement.

Les locomotrices à voyageurs peuvent réaliser leur puissance continue à la vitesse de 50 kilomètres à l'heure et la maintenir par le shuntage des inducteurs jusqu'à 74 kilomètres à l'heure. Pour les locomotives à marchandises, les chiffres ci-dessus sont respectivement de 30 et 45 kilomètres à l'heure.

Toutes ces machines sont munies du freinage, par récupération qui permet de relenir à la descente le train qu'elles penvent remorquer à la montée sur la même déclivité. Elles sont munies du contrôle série parallèle à contacteurs et arbre à cames et disposées pour la commande par unités multiples.

Les services auxiliaires sont assurés au moyen d'un groupe moteur-générateur de 72 kilowatts comprenant un moteur alimenté à 1.500 volts qui actionne d'une part une génératricè à 120 volts, d'autre part une génératrice à tension variable pour lexcitation des moleurs pendant la récupération et enfin les 2 ventilateurs des moteurs.

50 locomotives de ce type sont actuellement en construction et les premières d'entre elles entreront en service dans le courant de la présente amnée.

En dehors de ces machines, la Compagnie du Midi a mis à l'étude pour le service des trains express des loconiotives de grande vilesse d'un type entièrement nouveau pouvant atteindre 120 kilomètres à lheure. Deux exemplaires en ont élé mis en construction à titre d'essai et seront livrés dans quelques mois.

\title{
Services que l'Agriculture et la Houille Blanche peuvent attendre du Reboisement.
}

\author{
Par M. PAUL DESCOMBES, Ingênieur en chef honoraire des Manufactures de l'Etat.
}

L'agriculture rançaisè a beaucoup pâti de la sécheresse en 1921. dous ses nécoltes comme dans son betail, et l'industrie de Is houille blanche a sourfert êgalement de l'insuffisance des eaux. Cittle rarete des exux provient en grande partie de ce que les monlaghus swnt insulfisamment boisëes : et les enseignements de 1921. qui viemnent conlimmer cenx de la guerre, ne doivent pas pine perdus de vur.

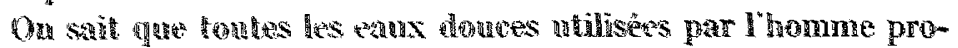
vinument de latmosphène, soil swus forme de pluies ou neiges mesurables par les plowionnetrn's, soil swus forme de condensations

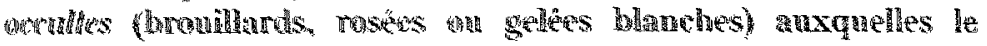

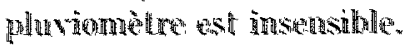

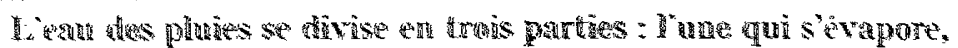

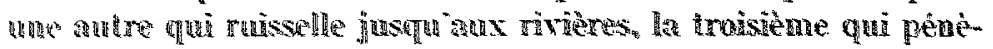

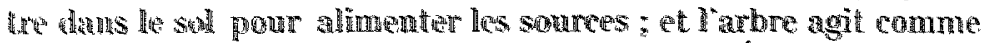
regulateur des eanx, en dimimuant lo ruissellemed pow aug-

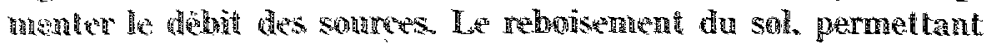

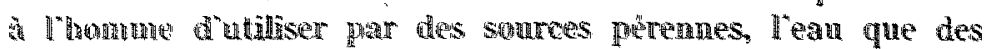

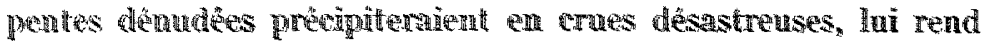

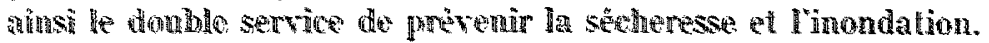

De son côté, l'eau du brouiliard et des rosées se dépose bien plus abondamment sur les arbres que sur le sol nu. Le reboisement, fournissant à l'homme un supplément d'eaux utilisables, lui donne de la sorte une seconde défense contre la sécheresse et lui permet de renforcer les débits d'étiage avec des eaux supplémentaires qui ne font courir aucun risque d'inondation,

\section{$*^{*} *$}

Il convient d'examiner ici dans quelle mesure le reboisement produit ce renforcement du débit.

La proportion de ce renforcement serait sans doute universellement connue depuis longtemps, si les expériences que I'Ingénieur en chef Conte-Grandchamps résumait dans son rapport du 10 janvier 1862 avaient été continuées: Nous avons fait, disait-il, dans le département de la Loire, pendant trois années consécutives, des expériences tendant à établir l'influence des forêts de sapins sur le débit des sources. Ces expériences nous ont démontré que, dans les terrains granitiques situés à 1.000 mètres environ au-dessus du niveau de la mer, le débit des sources est deux fois plus considérable dans les terrains boisés que dans 
les terrains déboisés. "Malheureusement ce rapport est resté jusqu'à l'année dernière enfoui dans descartons.

Cette question pouvail donc être considérée comme absolument nouvelle quand l'Association centrale pour l'Aménagement des montagnes en entreprit l'étude en 1904.

L'enquête ouverte par une note du 4 mars 1904, "Sur un mode de l'action hydrologique des forêts ", fit recueillir d'utiles données qui furent résumées le 8 décembre 1919 dans une communication à l'Académie des Sciences " Sur le concours des arbres pour soutirer de l'eau à l'atmosphère ", et développée dans un mémoire, l'Influence du reboisement sur les condensations occultes.

Une discussion du 23 mars à l'Académie d'Agriculture, à laquelle ont pris part MM. Dabat, Angot et Hickel, a fait ressortir combien l'action de l'arbre sur les condensations occultes es! supérieure à cellè des herbages, comment aussi s'exerce l'action spéciale des lisières et des rideaux d'arbres ; mais il n'est pas uncore possible de calculer dans quelle proportion le supplément d'eau fourni par les condensations occultes se distribue entre l'évaporation et le renforcement des cours d'eau. Cette proporlion est d'ailleurs très variable suivant les bassins de réceplion et, dans ces conditions, les recherches ont dû s'orienter vers la comparaison des débits évacués par des bassins similaires ne différant que par le taux de boisement, ou de bassins dont on connaît à la fois le débit et la situation pluviométrique.

Comme comparaison de bassins similaires, celui de la Pique, boisé à $40 \%$ débite annuellement $1,732.000$ mètres cubes par kilomètre carré, tandis que le bassin contigu de l'Onne, boisé à $5 \%$ en débite seulement 693.000 ; et on a calculé d'après ces données que, dans la vallée de Luchon, un hectare boisé fournit au déversoir cinq tois plus d'eau qu'un hectare déboisé.

Pour des bassins débitant plus d'eau qu'ils n'en reçoivent des pluies ou neiges, des calculs du même genre montrent que le débit par hectare boisé se trouve quadruplé dans celui du lac Léman et doublé dans le bassin de réception du réservoir de la Nouche (plateau de Langres).

On savait déjà par les expériences de Comle-Grandchamps que dans la région de Furens (Loire) le débit des sources est deux fois plus considérable dans les terrains boisés ; et ces déterminations, quoique peu nombreuses, font voir que le reboisement renforce les débits dans une proportion variable du double au quintuple suivant les régions.

Elles permettent d'apprécier l'importance du renforcement que le reboisement peut apporter aux ressources hydrauliques et de l'utiliser sans attendre le résultat des expériences de longue haleine qui sont entreprises à son sujet.

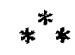

Une première série d'expériences est en cours, avec la collaboration de l'Administration forestière, pour déterminer en diverses stations les quantités d'eau déposées par les condensations occultes sur des récepteurs identiques et sur des végétaux variés, exposés dans les mêmes conditions; elles fourniront: d'ici quelques années des données numériques indispensables.

Une seconde série d'expériences est nécessaire aussi pour déterminer le renforcement utilisable des débits dans un même bassin dont on aura fait varier le revêtement végétal, d'après la méthode que Cézanne préconisait dẻs 1872 en disant : "On ne doit pas comparer des bassins distincts, mais les états successifs du même bassin $\%$. L'éminent ingénieur signalait ainsi l'unique moyen de démontrer incontestablement l'effet régulateur des forêts, et cette démonstration serait depuis longtemps acquise si l'expérience avaít été commencée dès cette époque. Tant qu'elle sera différée, les apôtres du reboisement resteront désarmés contre des affirmations renversantes, d'après les- quelles " la forêt aggraverant l'inondation au lieu de l'atténuer ", ou bien " le reboisement priverait d'eau les régions insuffisamment arrosées par les pluies ". L'intérêt de cette coûteuse expérience étant aujourd'hui doublé par la nécessité des constatations relatives au renforcement des débits par la forêt, l'Association centrale pour l'Aménagement des montagnes s'occupera de réunir des ressources pour l'exécuter dans la vallée d'Ossau, sur un des territoires qu'elle a déjà mis à l'abri du bétail.

$$
*^{*} *
$$

Le volume des eaux utilisables ne peut pas être instaniancment renforcé, mais les nouveaux reboisements produiront leur plein effet hydrologique quand les arbres auront pris un développement suffisant, quinze ou vingt ans après leur plantation, la rapidité du renforcement variant d'ailleurs avec les essences employées comme avec la fertilité du sol reboisé, et le début du renforcement ne se fera pas attendre aussi longtemps.

D'après les expériences effectuées dans les Pyrénées et les Alpes par l'Association centrale pour l'Aménagement des montagnes, il suffit d'évincer pendanl cinq ans les chèvres et les moutons étrangers, avec une dépense totale de 5 francs par hectare, pour faire reparaître des bois insoupconnés et pour embroussailler jusqu'aux rochers, sans privation ni gêne pou les habitanis et leurs troupeaux.

L'embroussaillement de la moilié de la surface permettrail d'obtenir à peu de frais et sans diminution sensible des ressources fourragères, au bout d'une période de cinq ans, un supplément d'eaux atmosphériques équivalant à $40 \%$ de la lame pluviale qui renforcerait d'autant le débit des eaux courantes. Ce genre d'embroussaillement constituerait une réparation sommaire, analogue à celle des bâtiments non habités que l'entretien des toitures et fermetures préserve des intempéries jusqu'au moment où leur utilisation motivera une réparation complète, et serait d'ailleurs bien plus efficace pour les montagnes que pour les bâtiments parce que la végétalion travaille sans relâche à consolider leur sol. Cette réparation sommaire aurait dâ s'appliquer en France aux 3 millions d'hectares dont Demontzey constatait la dégradation en 1889 ; si l'on avait fait aussitôt une dépense d'environ 15 millions pour arrêter la marche accélérée de la dégradation, l'agriculture et la houille blanche seraient actuellement bien mieux pourvues d'eau, et la seule atténuation des pertes causées par la sécheresse exceptionnelle de 1921 compenserait largement les frais de cet embroussaillement.

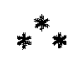

Il est indispensable de régénérer au plus vite l'armure végétale de la France pour régulariser et renforcer le régime des eaux dans l'intêrêt de l'agriculture, des forces motrices et de la navigation. L Administration des Eaux et Forêts y consacre depuis longtemps sa science et son inlassable dévouement, mais l'Etat ne peut pas tout faire. Après les enseignements de la sécheresse comme après ceux de la guerre, après la terrible saignée que viennent de subir les forèts françaises, les initiatives et les capitaux doivent collaborer à la régénération sylvo-pastorale, cet élément essentiel de la prospérite nationale.

\section{J'al l'honneur de vous proposer le vou :}

"Que l'Etat favorise énergiquement par ses exemples, par * son enseignement, par ses appuis matériels et moraux, par a ses immunités fiscales et par l'adaptation de la législation " au concours des capitaux collectifs ou particuliers, le maintien a et l'amélioration des forêts existantes, l'aménagement sylvo4astor. 1 des montagnes et le reboisement des surfaces dénu* dées." 